\title{
Numerical Method for the Solution of the One-Dimensional Anomalous Subdiffusion Equation with a Variable Diffusion Coefficient
}

\author{
M. BŁASIK* \\ Częstochowa University of Technology, al. Armii Krajowej 19, 42-201 Częstochowa, Poland \\ Doi: $10.12693 /$ APhysPolA.138.228 \\ *e-mail: marek.blasik@gmail.com
}

\begin{abstract}
In this paper we present a numerical solution of a one-dimensional anomalous subdiffusion equation with a variable diffusion coefficient. The proposed method is an extension of the fractional Crank-Nicolson method for a subdiffusion equation with constant diffusion coefficient. The work of the proposed algorithm is illustrated by the results of numerical simulations.

topics: fractional derivatives and integrals, fractional partial differential equations, numerical methods, transport processes
\end{abstract}

\section{Introduction}

Diffusion is a physical process in which substances mix due to the random motion of their molecules from a region of high concentration to one of lower concentration. As a result of collisions between molecules of the diffusing substance and solvent molecules, they move in accordance with the relationship $\left\langle x^{2}(t)\right\rangle \sim t$ (Brownian motions), where $\left\langle x^{2}(t)\right\rangle$ is a mean squared displacement of the diffusing molecule in the course of time $t$.

However, it turns out that many phenomena occurring in nature [1-3] can be described by a more general relationship $\left\langle x^{2}(t)\right\rangle \sim t^{\alpha}$ that leads to the anomalous diffusion equation.

Voller et al. [4] considered a more general model of anomalous heat diffusion with a variable diffusion coefficient $x^{(2 \beta-1) / \beta}$, which leads to the relationship $\left\langle x^{2}(t)\right\rangle \sim t^{2 \alpha \beta}, \alpha, \beta \in(0,1]$. The variable diffusion coefficient has its physical justification in sediment mass transport [5].

The numerical method discussed in this paper is an extension of the generalized Crank-Nicolson method [6] for an anomalous diffusion equation with a variable diffusion coefficient.

\section{Preliminaries}

Definition 1: The left-sided Riemann-Liouville integral of order $\alpha$, denoted as $I_{0+}^{\alpha}$, is given by the following formula for $\operatorname{Re}(\alpha)>0$ :

$$
I_{0+}^{\alpha} f(t)=\frac{1}{\Gamma(\alpha)} \int_{0}^{t} \frac{f(u) \mathrm{d} u}{(t-u)^{1-\alpha}},
$$

where $\Gamma$ is the Euler gamma function.
Definition 2: Let $\operatorname{Re}(\alpha) \in(0,1]$. The left-sided Caputo derivative of order $\alpha$ is given by the formula

$$
{ }^{C} D_{0+}^{\alpha} f(t)= \begin{cases}\frac{1}{\Gamma(\alpha)} \int_{0}^{t} \frac{f(u) \mathrm{d} u}{(t-u)^{1-\alpha}}, & 0<\alpha<1, \\ \frac{\mathrm{d} f(t)}{\mathrm{d} t}, & \alpha=1 .\end{cases}
$$

Property 3: Let function $f \in C^{1}(0, b)$. Then, the composition rule for the left-sided RiemannLiouville integral and the left-sided Caputo derivative is given as follows:

$$
I_{0+}^{\alpha C} D_{0+}^{\alpha} f(t)=f(t)-f(0) .
$$

Definition 4: Let $\Pi=\{(x, t): x \in(0, l) ; t \in$ $\left.\left(0, t^{*}\right)\right\}$ be a continuous region of solutions for the partial differential equation and $t^{*}$ be a fixed value of variable $t$. Then the set

$$
\begin{gathered}
\Pi^{*}=\left\{\left(x_{i}, t_{j}\right) \in \Pi: x_{i}=i \Delta x, i \in\{0, \ldots m\},\right. \\
\left.\Delta x=\frac{l}{m} ; t_{j}=j \Delta t, j \in\{0, \ldots n\} ; \Delta t=\frac{t^{*}}{n}\right\}
\end{gathered}
$$

is called the rectangular regular mesh described by the set of nodes.

Definition 5: Let $\gamma>-1, \delta \in C, z \in C$. The twoparameter Wright function is given by the following series:

$$
W(z ; \gamma, \delta)=\sum_{k=0}^{\infty} \frac{z^{k}}{k ! \Gamma(\gamma k+\delta)} .
$$

\section{Mathematical formulation} and numerical solution of the problem

Consider the following subdiffusion equation with a variable diffusion coefficient

$$
{ }^{C} D_{0+, t}^{\alpha} u(x, t)=\frac{\partial}{\partial x}\left(x^{\frac{2 \beta-1}{\beta}} \frac{\partial}{\partial x} u(x, t)\right),
$$

supplemented with Dirichlet boundary conditions

$$
u(0, t)=u_{0}, \quad u(l, t)=u_{l}, \quad t>0,
$$


and initial condition

$$
u(x, 0)=u_{l}, \quad x>0 .
$$

The diffusion coefficient is a power function of the spatial variable $x$, so Eq. (4) can be written in the following form:

$$
\begin{aligned}
& { }^{C} D_{0+, t}^{\alpha} u(x, t)=\frac{2 \beta-1}{\beta} x^{\frac{\beta-1}{\beta}} \frac{\partial}{\partial x} u(x, t) \\
& +x^{\frac{2 \beta-1}{\beta}} \frac{\partial^{2}}{\partial x^{2}} u(x, t) .
\end{aligned}
$$

Integrating Eq. (7) using the left-sided RiemannLiouville integral we get the following equation:

$$
\begin{aligned}
& I_{0+, t}^{\alpha}{ }^{C} D_{0+, t}^{\alpha} u(x, t)=\frac{2 \beta-1}{\beta} x^{\frac{\beta-1}{\beta}} I_{0+, t}^{\alpha} \frac{\partial}{\partial x} u(x, t) \\
& \quad+x^{\frac{2 \beta-1}{\beta}} I_{0+, t}^{\alpha} \frac{\partial^{2}}{\partial x^{2}} u(x, t) .
\end{aligned}
$$

Using Property 3 to Eq. (8), we obtain the integrodifferential equation in the form of

$$
\begin{aligned}
& u(x, t)=u(x, 0)+\frac{2 \beta-1}{\beta} x^{\frac{\beta-1}{\beta}} I_{0+, t}^{\alpha} \frac{\partial}{\partial x} u(x, t) \\
& +x^{\frac{2 \beta-1}{\beta}} I_{0+, t}^{\alpha} \frac{\partial^{2}}{\partial x^{2}} u(x, t) .
\end{aligned}
$$

We discretize Eq. (9) approximating: the first-order derivative with the differential quotient

$$
\left(\frac{\partial u}{\partial x}\right)_{i, j}=\frac{u_{i+1, j}-u_{i-1, j}}{2 \Delta x}+h\left((\Delta x)^{2}\right),
$$

the second order derivative with the differential quotient

$$
\left(\frac{\partial^{2} u}{\partial x^{2}}\right)_{i, j}=\frac{u_{i+1, j}-2 u_{i j}-u_{i-1, j}}{(\Delta x)^{2}}+h\left((\Delta x)^{2}\right) .
$$

The left-sided Riemann-Liouville integral is approximated by the fractional trapezoidal rule

$$
I_{0+, t}^{\alpha} u\left(x_{i}, t_{k+1}\right) \approx \sum_{j=0}^{k+1} c_{j, k+1} u_{i, j},
$$

where

$$
\begin{aligned}
& c_{j, k+1}=\frac{(\Delta t)^{\alpha}}{\alpha(\alpha+1) \Gamma(\alpha)} \\
& \times\left\{\begin{array}{cc}
\left(k^{\alpha+1}-(k-\alpha)(k+1)^{\alpha}\right) & \text { for } j=0, \\
\left((k-j+2)^{\alpha+1}+(k-1)^{\alpha+1}\right. & \\
\left.-2(k-j+1)^{\alpha+1}\right) & \text { for } 1 \leq j \leq k, \\
1 & \text { for } j=k+1 .
\end{array}\right.
\end{aligned}
$$

Finally, we obtain the implicit numerical scheme

$$
\begin{aligned}
& u_{i+1, k+1}\left(-w_{i, k+1, k+1}^{1}-w_{i, k+1, k+1}^{2}\right) \\
& \quad+u_{i, k+1}\left(1+2 w_{i, k+1, k+1}^{2}\right) \\
& \quad+u_{i-1, k+1}\left(w_{i, k+1, k+1}^{1}-w_{i, k+1, k+1}^{2}\right)=u_{i, 0} \\
& \quad+\sum_{j=0}^{k} u_{i+1, j}\left(w_{i, j, k+1}^{1}+w_{i, j, k+1}^{2}\right)-2 w_{i, j, k+1}^{2} u_{i, j} \\
& \quad+u_{i-1, j}\left(w_{i, j, k+1}^{2}-w_{i, j, k+1}^{1}\right)
\end{aligned}
$$

where the weights $w_{i, j, k+1}^{1}$ and $w_{i, j, k+1}^{2}$ are defined in the following form:

$$
\begin{aligned}
& w_{i, j, k+1}^{1}=\frac{(2 \beta-1) c_{j, k+1}}{2 \beta \Delta x} x_{i}^{\frac{\beta-1}{\beta}}, \\
& w_{i, j, k+1}^{2}=\frac{c_{j, k+1}}{(\Delta x)^{2}} x_{i}^{\frac{2 \beta-1}{\beta}} .
\end{aligned}
$$

The numerical method (13) can be written as a system of linear equations in the matrix form

$$
\boldsymbol{A} \boldsymbol{U}_{k+1}=\boldsymbol{B},
$$

where $\boldsymbol{A}$ and $B$ are defined as follows:

$$
\boldsymbol{A}=\left[\begin{array}{cccccccc}
a^{2} & a^{3} & 0 & 0 & \cdots & 0 & 0 & 0 \\
a^{1} & a^{2} & a^{3} & 0 & \cdots & 0 & 0 & 0 \\
0 & a^{1} & a^{2} & a^{3} & \cdots & 0 & 0 & 0 \\
\vdots & \vdots & \vdots & \vdots & \ddots & \vdots & \vdots & \vdots \\
0 & 0 & 0 & a^{1} & a^{2} & a^{3} & 0 & 0 \\
\vdots & \vdots & \vdots & \vdots & \ddots & \vdots & \vdots & \vdots \\
0 & 0 & 0 & 0 & \cdots & a^{1} & a^{2} & a^{3} \\
0 & 0 & 0 & 0 & \cdots & 0 & a^{1} & a^{2}
\end{array}\right]
$$

$$
\boldsymbol{B}=\left[\begin{array}{c}
b_{1}-a^{1} u_{0, k+1} \\
b_{2} \\
b_{3} \\
\vdots \\
b_{i} \\
\vdots \\
b_{m-2} \\
b_{m-1}-a^{3} u_{m, k+1}
\end{array}\right] \text {. }
$$

The elements of matrices $\boldsymbol{A}$ and $\boldsymbol{B}$ are defined by the formulas

$$
\begin{aligned}
a^{1} & =w_{i, k+1, k+1}^{1}-w_{i, k+1, k+1}^{2} \\
a^{2} & =1+2 w_{i, k+1, k+1}^{2} \\
a^{3} & =-w_{i, k+1, k+1}^{1}-w_{i, k+1, k+1}^{2} \\
b_{i} & =u_{i, 0}+\sum_{j=0}^{k} u_{i-1, j}\left(w_{i, j, k+1}^{2}-w_{i, j, k+1}^{1}\right) \\
& -2 u_{i, j} w_{i, j, k+1}^{2}+u_{i+1, j}\left(w_{i, j, k+1}^{1}+w_{i, j, k+1}^{2}\right) .
\end{aligned}
$$

\section{Numerical examples}

We will now present the results of three numerical simulations to demonstrate the proposed method. In all considered cases, the following parameters of the mathematical model and the rectangular mesh were adopted: $u_{0}=1, u_{l}=0, l=10, \Delta t=0.005$, $\Delta x=0.2$. In addition, the first simulation also assumed $\alpha=1$ and $\beta=0.6$.

Figure 1 presents the numerical solution to the initial-boundary value problem formulated by Eqs. (4)-(6), which is consistent with the solution of the model described by the partial differential equation with derivatives of integer order with a non-linear flux (we observe super diffusive behavior because $\alpha \beta=0.6)$. 


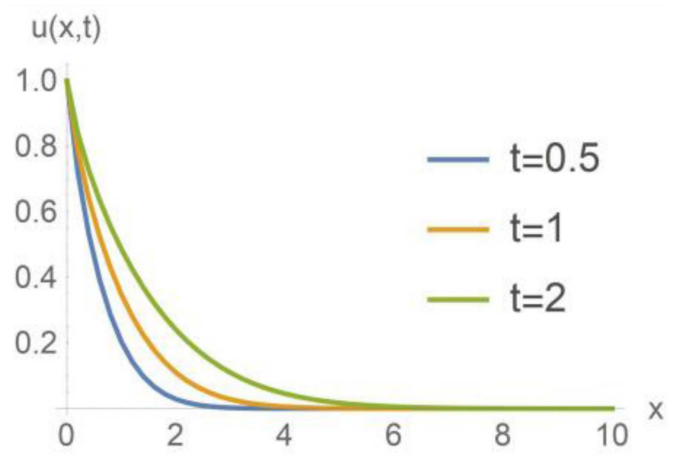

Fig. 1. Numerical solution of the initial-boundary value problem for $\alpha=1$ and $\beta=0.6$.

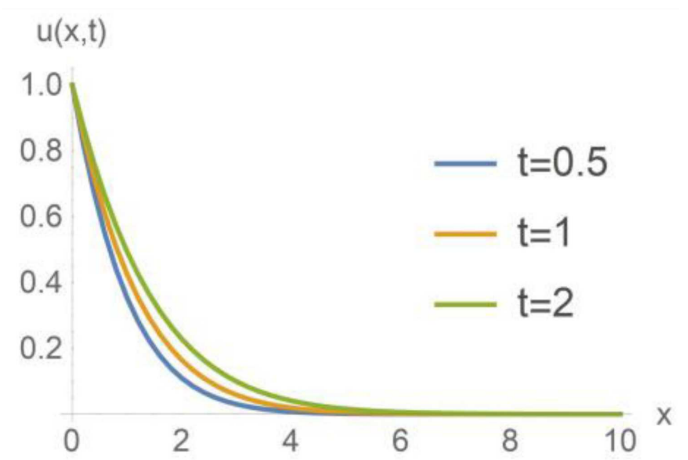

Fig. 2. As in Fig. 1, but for $\alpha=0.53$ and $\beta=0.5$.

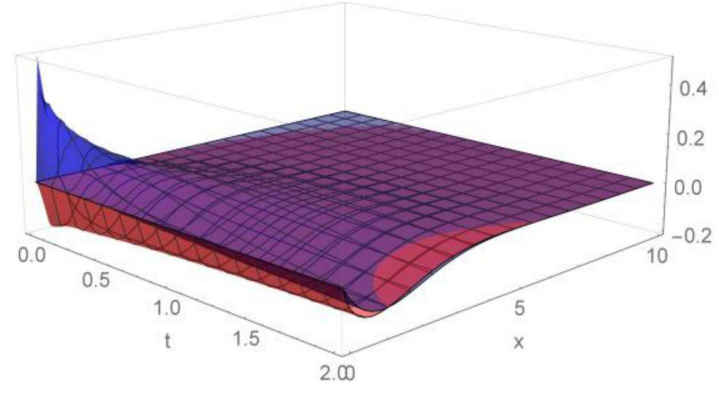

Fig. 3. Comparison of the results of the first and second simulation with the solution of the parabolic equation $(\alpha=1$ and $\beta=0.5)$.

In the second simulation, the parameters $\alpha=$ 0.53 and $\beta=0.5$ were selected so as to obtain an equivalent solution (areas under the curves for $t=2$ in Figs. 1 and 2 are almost equal) to the solution from the first simulation. The received numerical results are shown in Fig. 2. The choice of parameters indicates that the solved equation is an anomalous diffusion equation with a constant diffusion coefficient (we observe strong sub-diffusion behavior because $\alpha \beta=0.265)$.

Figure 3 presents a comparison of the results from the first and second simulation in relation of the results of the third simulation obtained for $\alpha=1$

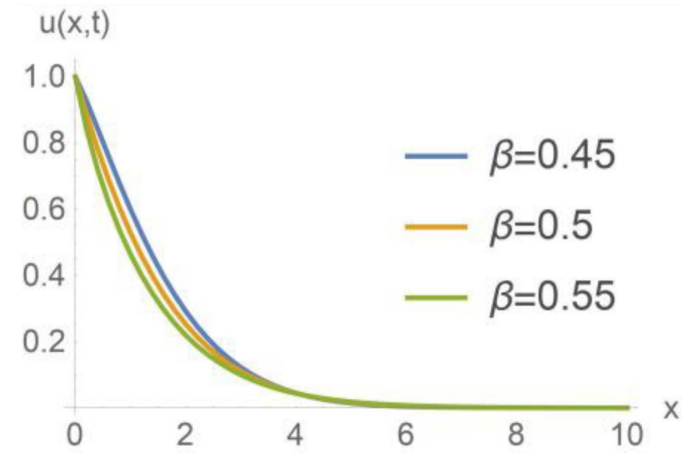

Fig. 4. As in Fig. 1, but for $\alpha=1$ and $\beta \in$ $\{0.45 ; 0.5 ; 0.55\}$.

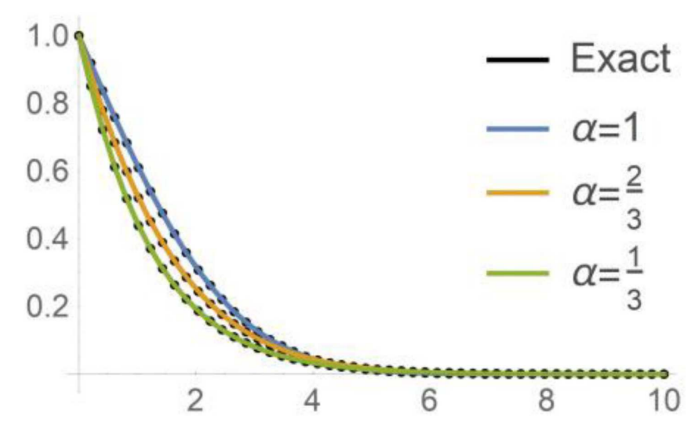

Fig. 5. As in Fig. 1, but for $\alpha \in\{1 / 3 ; 2 / 3 ; 1\}$ and $\beta=0.5$.

and $\beta=0.5$ (classical diffusion because $\alpha \beta=0.5$ ). In order to determine the surface of the red color, the difference of the solution from the first simulation and the solution from the third simulation was calculated at each point of the $\Pi^{*}$ mesh. The values between nodes have been interpolated. The surface marked in blue was created in the same way, as a difference of the solution from the second simulation and the solution from the third simulation at each point of the $\Pi^{*}$ mesh.

The solution obtained in the first simulation, despite the super diffusion nature, has values smaller than the solution in the third simulation. This is due to the special arrangement of boundary and initial conditions, for which a significant part of the non-zero solution is for $x \in(0,1)$, where $\sqrt[3]{x}<1$. The opposite behavior is observed for the solution in the second simulation (subdiffusion), which results directly from inequality $t<t^{0.53}$ for $t \in(0,1)$.

The results in Fig. 4 indicate that for the considered initial-boundary value problem modeled displacement of diffusive molecules is a decreasing function of the $\beta$ parameter. This observation results directly from the form of the diffusion coefficient for which we observe the relationship $x^{2 / 11}<$ $1<x^{2 / 9}$ for $x \in(0,1)$.

The closed analytical solution (only if $\beta=0.5$ ) expressed in terms of the Wright function

$$
u(x, t)=W\left(-\frac{x}{t^{\alpha / 2}} ;-\frac{\alpha}{2}, 1\right)
$$


obtained by Gorenflo et al. [7] allowed partial validation of the method proposed in Sect. 3. The results of the comparison are shown in Fig. 5 and allow us to state that in this particular case the numerical scheme works correctly.

\section{Conclusions}

In this paper, we developed a numerical scheme for solving a one-dimensional anomalous diffusion equation with a variable diffusion coefficient. The proposed method is an extension of the fractional Crank-Nicolson method. The problem of testing the stability of the method was not discussed in the paper and will be the subject of further research. However, it has been noticed that the method is not always stable and the unknown stability condition strongly depends on the parameter $\beta$.

\section{References}

[1] E.R. Weeks, J.S. Urbach, L. Swinney, Physica D 97, 291 (1996).

[2] T.H. Solomon, E.R. Weeks, H.L. Swinney, Phys. Rev. Lett. 71, 3975 (1993).
[3] T. Kosztołowicz, K. Dworecki, S. Mrówczyński, Phys. Rev. E 71, 041105 (2005).

[4] F. Falcini, R. Garra, V. Voller, Int. J. Therm. Sci. 137, 584 (2019).

[5] A.D. Howard, Water Resources Res. 30, 2261 (1994).

[6] M. Błasik, in: Proc. 23rd Int. Conf. on Methods \& Models in Automation \&6 Robotics, Miedzyzdroje (Poland), 2018, IEEE, p. 726.

[7] R. Gorenflo, Y. Luchko, F. Mainardi, J. Computat. Appl. Math. 118, 175 (2000). 